\title{
LAS TRES EDADES DE LA PRODUCCIÓN INDUSTRIAL en Le Creusot-Montceau
}

\section{Françoise Fortunet}

Université de Bourgogne

\section{RESUMEN}

En este artículo se analiza la evolución del imperio de la industria del carbón en las ciudades de Le Creusot y Montceau. Esta historia se inicia a partir de finales de 1780, cuando se descubre la gran calidad del carbón de Le Creusot para la producción de piezas de fundición. La accesibilidad que supone la navegación fluvial y el ferrocarril permitirá, en medio siglo, desarrollar un nuevo tipo de industria, concentrando materia prima, fuerza de trabajo y productos acabados. La consolidación del emporio industrial da lugar a la fundación de tres ciudades: Le Creusot, Montchanin y Montceau, cuyo desarrollo urbano sigue un patrón común, basado en la conexión directa entre residencia y lugar de trabajo, aunque resultan evidentes las precarias condiciones laborales y la segregación social. En los albores de la segunda guerra mundial, las nuevas ciudades alcanzan su apogeo económico y comienzan los primeros problemas entre la ciudad y la mina. La entrada en la etapa post-industrial supone la reestructuración urbana a partir del declive económico, la quiebra y disolución de la compañía minera y, finalmente, la recuperación patrimonial de "la cuenca carbonífera". 


\section{ABSTRACT}

This article examines the evolution of the coal industry in the cities of Le Creusot and Montceau. The story starts at the end of 1780 , with the they discovery of the Le Creusot high quality coal. Accessibility involving inland waterways and rail allow, in half a century, developing a new type of industry, concentrating raw material, labor and finished products. The consolidation of the industrial empire leads to the founding of three cities: Le Creusot, Montchanin and Montceau, whose urban development follows a common pattern, based on the direct link between residence and workplace, although combining precarious working conditions and social segregation. Just before World War II, the new cities reached its economic peak and begin the first problems between them and the mine. Post-industrial period implies urban restructuring affected by economic decline, bankruptcy and dissolution of the mining company and, finally, the restoration of "the coal basin".

\section{INTRODUCCIÓN}

Todo empieza con el descubrimiento de "...un paisaje desagradable con apariencia inusual", tal y como lo describe Arthur Young en 1.789, cuando visita la nueva fundición real en Montcenis. Al fines del siglo XVIII el entorno de Montceau no resulta en absoluto atractivo; de hecho es “... menos fértil que la cercana Charolais; más frío y pedregoso; un lugar con amplias extensiones sin cultivar, terrenos en barbecho donde crecen brezos y zarzas".

Aún así muchos expertos, tanto franceses como extranjeros, visitan la zona; científicos como el ingles Wilkinson, y algunos fabricantes, como Ignace de Wendel, maestro herrero de Lorraine, que viene a analizar el carbón de Le Creusot, extraído de los terrenos entregados a Françoise de la Chaise en 1769. Este carbón demuestra tener la calidad necesaria para producir piezas de fundición según el método inglés. 
La moderna epopeya industrial empieza en una hondonada, donde en 1782 la compañía de "La Fonderie de Montcenis et d'Indret" levanta una fundición bajo la protección real. En 1786 se añade una fábrica de vidrio para alojar la Manufactura de Cristales de la Reina, hasta entonces localizada en Sèvres.

Como no existe ninguna vía adecuada para transportar materias primas ni productos manufacturados, el Estado de Borgoña decide en 1782 construir un canal entre los ríos Saône y Loire, al objeto de unir el Mediterráneo con el Atlántico y llegar a la capital a través del canal Briare. Emiland Gauthey, ingeniero jefe, dirige el proyecto, y de este modo empieza a principios de 1793 la navegación a través de los canales Central y Charolais. La fundición se conecta al canal, al inicio a través de transporte animal, y más tarde con un ferrocarril privado. Medio siglo más tarde, en 1860, se realiza el empalme con el ferrocarril hasta París y se levantan estaciones en Le Creusot y Montceau. Ambos asentamientos responden a un nuevo concepto de industria, una factoría completa, que aloja los diversos estadios productivos en diferentes edificios, y concentra en un mismo lugar materia prima, fuerza de trabajo y productos acabados. Constituye un modelo de factoría decimonónica, con grandes naves para acoger maquinaria bien diversa, mano de obra abundante y el carbón de los yacimientos cercanos.
Montceau-les-Mines: Vista general de la ciudad, el puerto y las minas de carbón. 1864 Original de impresión fotográfica montada en 2 partes. Colección privada

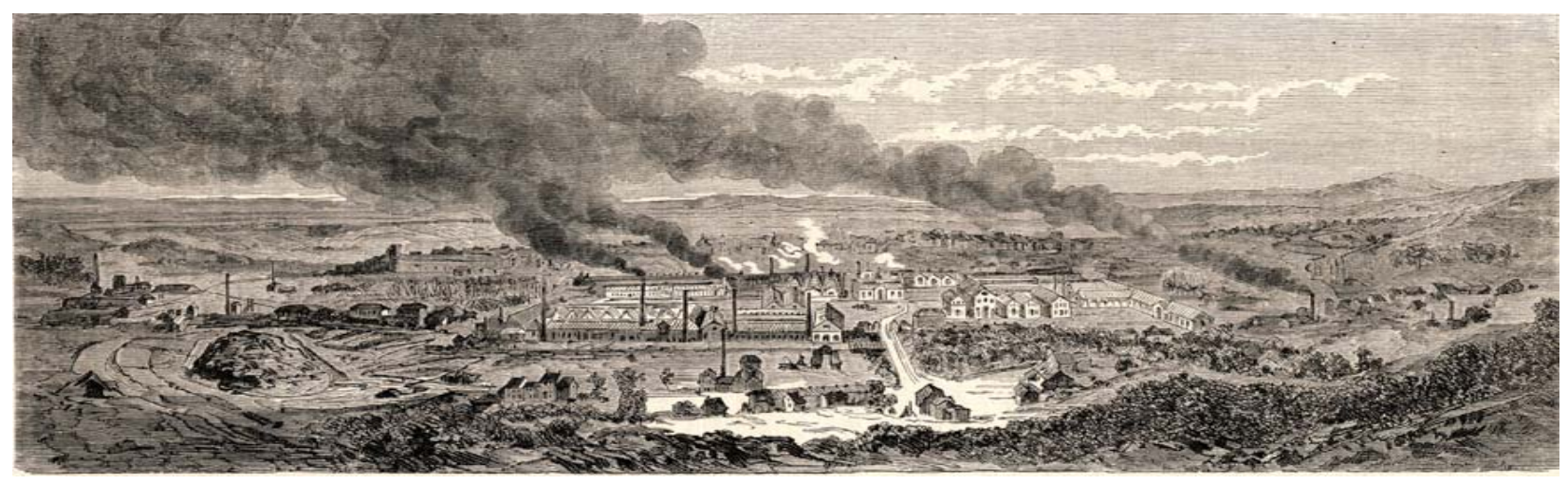


Los inicios tienen mucho de novedad, y a esto se añaden las dificultades debidas al contexto político. A pesar de trabajar para el ejército, la siderurgia de Le Creusot se ve afectada durante medio siglo por una sucesión de bancarrotas y compraventas. La industria extractiva se limita a las minas de carbón, aunque los propietarios pretenden imprimir un nuevo carácter a la compañía. En 1820 se levantan las primeras grandes naves, características de la nueva arquitectura industrial, a las que seguirá, en 1825, un conjunto residencial para obreros siguiendo modelos ingleses. Este grupo, Combe des Mineurs, constituye una inflexión en la evolución de la vivienda obrera, ya que incluye casas unifamiliares en lugar de bloques o hileras de viviendas, opción característica hasta entonces en Le Creusot y en tantos otros lugares.

Le Creusot: Establecimientos Schneider - fábrica de Schneider en Le Creusot en 1847 A partir de Lanzarote $F$. Bonhomme.

Parte de una "fábrica de Panorama Schneider en Le Creusot, 20 años de diferencia"

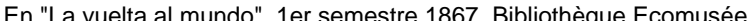

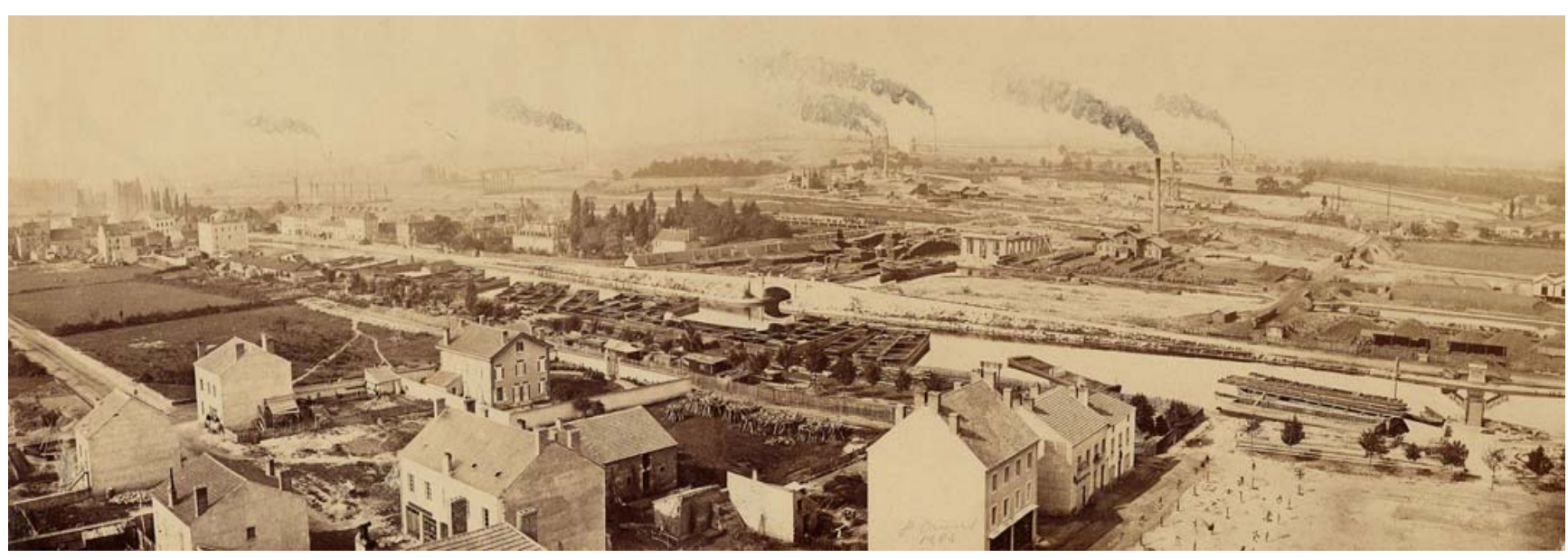




\section{LA CONSTRUCCIÓN DE UN EMPORIO INDUSTRIAL}

Dos acontecimientos marcan el arranque de una nueva era industrial. En 1832 se produce la división en dos de la concesión minera y la separación de las minas de la manufactura siderúrgica de Le Creusot. Jules Chagot, antiguo propietario de Le Creusot, crea su propia compañía y en 1836 los hermanos Eugene y Adolph Schneider adquieren la compañía de Le Creusot. Con ello arranca la construcción de un nuevo emporio industrial, que llega a ser la primera compañía siderúrgica francesa al final del Segundo Imperio.

El desarrollo de estas actividades da lugar a la fundación de tres ciudades, Le Creusot en 1793, Montchanin en 1854 y Montceau en 1856. Cada una de ellas viene marcada por una producción característica. $Y$ si bien Le Creusot y Montceau son totalmente diferentes, tienen algunos elementos en común. Hasta los albores de la primera Guerra Mundial se caracterizan por su notable crecimiento económico, incremento de la población y desarrollo urbano, que les lleva a convertirse en las principales ciudades del departamento de Saône et Loire. Los 2.300 habitantes de Le Creusot en el censo de 1836 y los 1.640 de la futura ciudad de Montceau en 1856, se incrementan hasta 30.500 en 1901 y 35.000 en 1910 en Le Creusot, y 28.500 en Montceau.

En ambos casos el desarrollo urbano sigue idénticos patrones funcionales, a medida que avanza la construcción de talleres y la explotación de nuevos yacimientos. Se puede reconocer un mismo esquema de viviendas para obreros cerca de las fábricas al objeto de evitar largos desplazamientos, asegurando una conexión directa entre hogares y lugar de trabajo, constituyendo las viviendas un lugar de descanso y privacidad. Ambas ciudades construyen con distinto ritmo las dotaciones públicas y privadas necesarias para dar respuesta a las demandas de la población obrera: escuelas, hogar infantil, casas para trabajadores ancianos, enfermerías y hospitales. Esto muestra una preocupación por las condiciones laborales, y es reconocido como modelo de organización en la Exposición Universal.

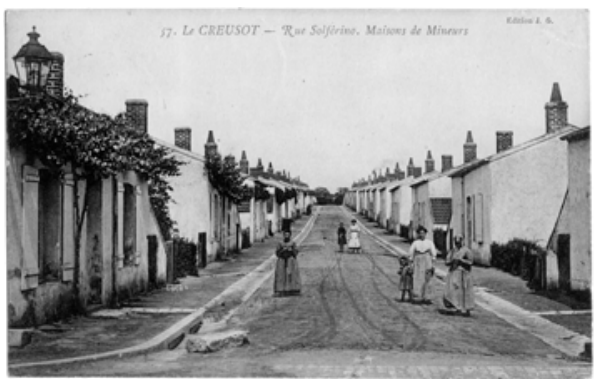

"17 - Le Creusot - Calle Solferino, las casas de los mineros" - al. J.B. Ciudad Villedieu, también llamada la ciudad de los menores

Postal, siglo 20. Collection Écomusée

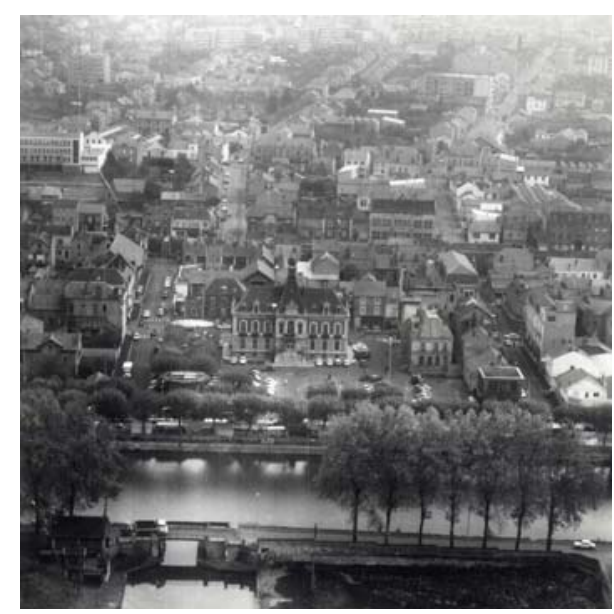

Montceau-les-Mines: Vista del centro de la ciudad, con el primer plano del Canal du Centre. En el centro el hotel de la ciudad, al fondo el área de Bellevue y a la izquierda del colegio Jean Moulin - sin fecha.

Collection Écomusée 
En 1867 la compañía Schneider Le Creusot gana el recién creado premio de Economía Social con un modelo de ciudad minera y en 1893 es la compañía Chagot la distinguida por sus logros sociales.

El plan de desarrollo urbano muestra una segregación social y espacial similar en ambas ciudades, separando las viviendas obreras de las del resto de empleados e ingenieros. Aún así, se pueden reconocer ciertas diferencias que persisten hasta hoy entre ambos núcleos. Le Creusot es una "isla industrial" de cerca de 4 kilómetros de extensión, rodeada de distritos construidos con los nuevos equipamientos y las innovaciones técnicas de la compañía durante el siglo XIX. El resultado es una ciudad sin un verdadero centro, más allá de los edificios administrativos de la factoría, cercanos a los principales equipamientos públicos, oficinas de correos y ayuntamiento, que constituyen hoy su centro urbano.

En Montceau las construcciones administrativas y comerciales se proyectan alejadas del área minera, inadecuada para acoger usos urbanos. Se disponen confrontadas a ambos lados del Canal del Centro, principal ruta de comunicaciones. La "nueva" ciudad de Montceau se convierte en un ente autónomo con la elección del primer alcalde republicano en 1876. Arranca entonces una confrontación entre poderes, el de la ciudad y el de la mina, al que solo la elección del prohombre Jean Bouveri pone fin, después de un cuarto de siglo de largas huelgas.
Le Creusot: visión general de la ciudad y las fábricas (tomado de la montaña Crêtes) - sin fecha Impresiones fotográficas (3 piezas instaladas.) Colección privada

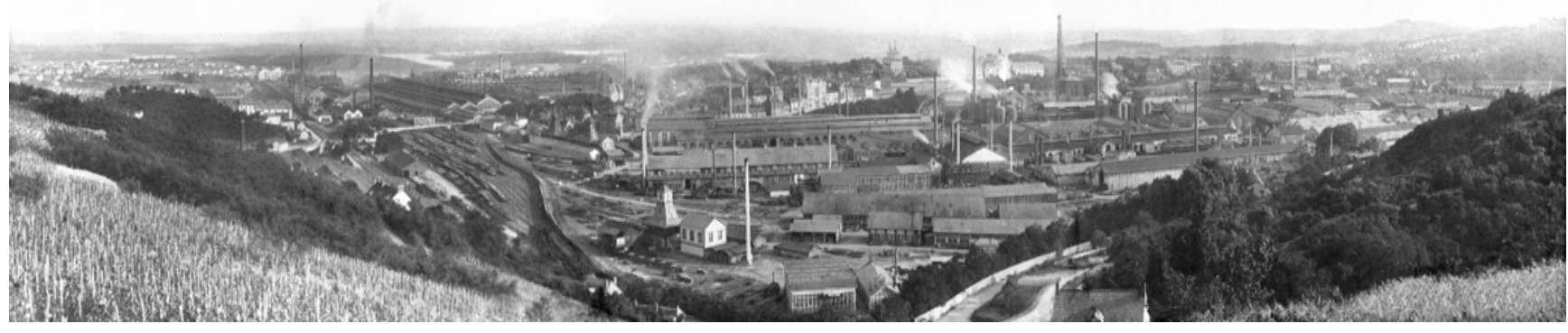


En los albores de la primera Guerra Mundial ambas ciudades alcanzan su apogeo como principales núcleos del departamento, constituyendo aún un atractivo centro generador de empleo, particularmente de mano de obra extranjera en el período de entreguerras.

\section{LA ENTRADA EN LA ETAPA POSTINDUSTRIAL}

El incremento de actividad debido a la recuperación económica tras la segunda Guerra Mundial y la constitución de la Comunidad Económica Europea, supone el inicio de un programa de reestructuración para ambas ciudades. Ello da lugar a una cierta ralentización de la economía, o incluso a la interrupción total de algunas actividades. El emporio de Le Creusot resulta el principal afectado, ya que después de la quiebra de la compañía en 1984, se produce la demolición de las áreas más antiguas, dando lugar a un extenso yermo industrial en la llanura de Riaux, donde antes se había levantado la cuna de la siderurgia francesa. De las demoliciones sistemáticas apenas se salvan unos pocos edificios, que se protegen como monumentos históricos. Se trata de las primeras viviendas obreras, construidas en 1825 y de los talleres de reparación de locomotoras y maquinaria, expresión de la primera arquitectura industrial.

Los yacimientos carboníferos resisten aún una década, gracias a la intervención pública, pero su fin se acerca, y el derrumbe de la bocamina cerca de la ciudad de Montceau marca el final del "territorio negro". En defensa de la cuenca carbonífera el Ayuntamiento inicia una política de recuperación de los espacios mineros. Dicha política adquiere pleno significado en Montceau, dotándole "de color" y permitiéndole encarar el siglo XXI, al convertir la vieja cantera en un espléndido bosque urbano de cien hectáreas.

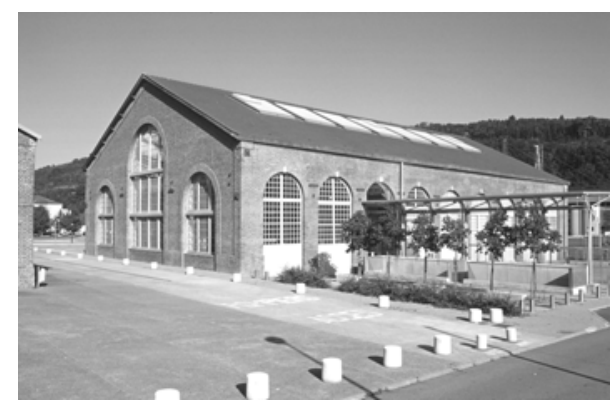

Le Creusot: Llano Riaux - Biblioteca de la Universidad (= antiguo edificio de "grúas y locomotoras"), vista general del exterior. 25/08/2000

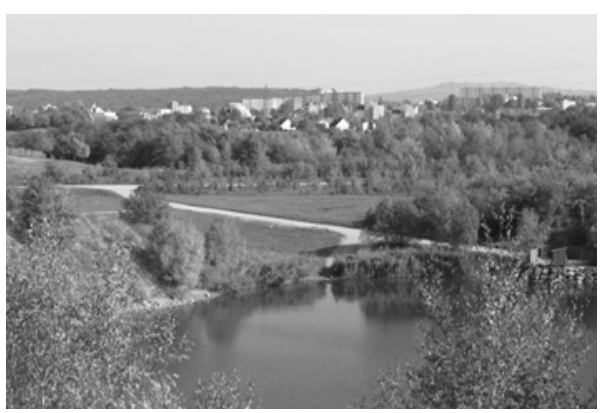

Montceau-les-Mines, Maugrand Saint-Pierre: Parque Minero - Estanque de Saint-Pierre Al fondo, la ciudad Montceau Daniel Busseuil cliché, 30/09/2003 